Commun. Korean Math. Soc. 27 (2012), No. 4, pp. 763-770

http://dx.doi.org/10.4134/CKMS.2012.27.4.763

\title{
LIGHTLIKE HYPERSURFACES OF A SEMI-RIEMANNIAN MANIFOLD OF QUASI-CONSTANT CURVATURE
}

\author{
DAE Ho JiN
}

\begin{abstract}
In this paper, we study the geometry lightlike hypersurfaces $(M, g, S(T M))$ of a semi-Riemannian manifold $(\widetilde{M}, \widetilde{g})$ of quasi-constant curvature subject to the conditions: (1) The curvature vector field of $\widetilde{M}$ is tangent to $M$, and (2) the screen distribution $S(T M)$ is either totally geodesic in $M$ or totally umbilical in $\widetilde{M}$.
\end{abstract}

\section{Introduction}

B. Y. Chen and K. Yano [2] introduced the notion of a Riemannian manifold of quasi-constant curvature as a Riemannian manifold $(\widetilde{M}, \widetilde{g})$ with the curvature tensor $\widetilde{R}$ satisfying the condition

$$
\begin{aligned}
\widetilde{g}(\widetilde{R}(X, Y) Z, W)= & \alpha\{\widetilde{g}(Y, Z) g(X, W)-\widetilde{g}(X, Z) g(Y, W)\} \\
& +\beta\{\widetilde{g}(X, W) \theta(Y) \theta(Z)-\widetilde{g}(X, Z) \theta(Y) \theta(W) \\
& +\widetilde{g}(Y, Z) \theta(X) \theta(W)-\widetilde{g}(Y, W) \theta(X) \theta(Z)\},
\end{aligned}
$$

where $\alpha, \beta$ are scalar functions and $\theta$ is a 1 -form defined by

$$
\theta(X)=\widetilde{g}(X, \zeta),
$$

and $\zeta$ is a unit vector field on $\widetilde{M}$ which called the curvature vector field of $\widetilde{M}$. It is well known that if the curvature tensor $\widetilde{R}$ is of the form (1.1), then $\widetilde{M}$ is conformally flat. If $\beta=0$, then $\widetilde{M}$ is a space of constant curvature.

A non-flat Riemannian manifold $\widetilde{M}$ of dimension $n(>2)$ is called a quasiEinstein manifold [1] if its Ricci tensor $\widetilde{R i c}$ satisfies the condition

$$
\widetilde{\operatorname{Ric}}(X, Y)=a \widetilde{g}(X, Y)+b \phi(X) \phi(Y),
$$

where $a, b$ are scalar functions such that $b \neq 0$ and $\phi$ is a non-vanishing 1-form such that $\widetilde{g}(X, U)=\phi(X)$ for any vector field $X$, where $U$ is a unit vector

Received July 5, 2011

2010 Mathematics Subject Classification. Primary 53C25, 53C40, 53C50.

Key words and phrases. totally geodesic, totally umbilical, lightlike hypersurface, semiRiemannian manifold of quasi-constant curvature. 
field. If $b=0$, then $\widetilde{M}$ is an Einstein manifold. It can be easily seen that every Riemannian manifold of quasi-constant curvature is a quasi-Einstein manifold.

The purpose of this paper is to study lightlike hypersurfaces of a semiRiemannian manifold of quasi-constant curvature. We prove two characterization theorems for lightlike hypersurfaces $(M, g, S(T M))$ of a semi-Riemannian manifold $(\widetilde{M}, \widetilde{g})$ of quasi-constant curvature:

- If $S(T M)$ is totally geodesic in $M$ and the curvature vector field $\zeta$ of $\widetilde{M}$ is tangent to $M$, then $\widetilde{M}$ and $M$ are flat manifolds (Theorem 3.3).

- If $S(T M)$ is totally umbilical in $\widetilde{M}$ and $\zeta$ is tangent to $M$, then $\widetilde{M}$ is a space of non-zero constant curvature $\alpha$ and $M$ is an Einstein manifold (Theorem 3.4).

\section{Lightlike hypersurface}

It is well known that the normal bundle $T M^{\perp}$ of the lightlike hypersurfaces $M$ of a semi-Riemannian manifold $(\widetilde{M}, \widetilde{g})$ is a subbundle of $T M$ of rank 1 . A complementary vector bundle $S(T M)$ of $T M^{\perp}$ in $T M$ is non-degenerate distribution on $M$, called a screen distribution on $M$, and

$$
T M=T M^{\perp} \oplus_{\text {orth }} S(T M),
$$

where $\bigoplus_{\text {orth }}$ denotes the orthogonal direct sum. We denote such a lightlike hypersurface by $M=(M, g, S(T M))$. Denote by $F(M)$ the algebra of smooth functions on $M$ and by $\Gamma(E)$ the $F(M)$ module of smooth sections of a vector bundle $E$ over $M$. It is well-known [4] that, for any null section $\xi$ of $T M^{\perp}$ on a coordinate neighborhood $\mathcal{U} \subset M$, there exists a unique null section $N$ of a unique vector bundle $\operatorname{tr}(T M)$ in $S(T M)^{\perp}$ satisfying

$$
\widetilde{g}(\xi, N)=1, \quad \widetilde{g}(N, N)=\widetilde{g}(N, X)=0, \forall X \in \Gamma\left(\left.S(T M)\right|_{\mathcal{U}}\right) .
$$

Then the tangent bundle $T \widetilde{M}$ of $\widetilde{M}$ is decomposed as follows;

$$
T \widetilde{M}=T M \oplus \operatorname{tr}(T M)=\left\{T M^{\perp} \oplus \operatorname{tr}(T M)\right\} \oplus_{\text {orth }} S(T M) .
$$

We call $\operatorname{tr}(T M)$ and $N$ the transversal vector bundle and the null transversal vector field of $M$ with respect to $S(T M)$ respectively.

Let $\widetilde{\nabla}$ be the Levi-Civita connection of $\widetilde{M}$ and $P$ the projection morphism of $\Gamma(T M)$ on $\Gamma(S(T M))$ with respect to the decomposition (2.1). For any vector fields $X, Y \in \Gamma(T M)$, the local Gauss and Weingartan formulas are given by

$$
\begin{aligned}
& \widetilde{\nabla}_{X} Y=\nabla_{X} Y+B(X, Y) N, \\
& \widetilde{\nabla}_{X} N=-A_{N} X+\tau(X) N, \\
& \nabla_{X} P Y=\nabla_{X}^{*} P Y+C(X, P Y) \xi, \\
& \nabla_{X} \xi=-A_{\xi}^{*} X-\tau(X) \xi,
\end{aligned}
$$

where $\nabla$ and $\nabla^{*}$ are the linear connections on $T M$ and $S(T M)$ respectively, $B$ and $C$ are the local second fundamental forms on $T M$ and $S(T M)$ respectively, $A_{N}$ and $A_{\xi}^{*}$ are the shape operators on $T M$ and $S(T M)$ respectively and $\tau$ is 
a 1-form on $T M$. Since $\widetilde{\nabla}$ is torsion-free, $\nabla$ is also torsion-free and $B$ is symmetric. From the fact that $B(X, Y)=g\left(\widetilde{\nabla}_{X} Y, \xi\right)$, we know that $B$ is independent of the choice of a screen distribution and satisfies

$$
B(X, \xi)=0, \quad \forall X \in \Gamma(T M) .
$$

The induced connection $\nabla$ of $M$ is not metric and satisfies

$$
\left(\nabla_{X} g\right)(Y, Z)=B(X, Y) \eta(Z)+B(X, Z) \eta(Y)
$$

for any $X, Y, Z \in \Gamma(T M)$, where $\eta$ is a 1-form such that

$$
\eta(X)=\widetilde{g}(X, N), \quad \forall X \in \Gamma(T M) .
$$

But the connection $\nabla^{*}$ on $S(T M)$ is metric. The above two local second fundamental forms of $M$ and on $S(T M)$ are related to their shape operators by

$$
\begin{array}{ll}
B(X, Y)=g\left(A_{\xi}^{*} X, Y\right), & \widetilde{g}\left(A_{\xi}^{*} X, N\right)=0, \\
C(X, P Y)=g\left(A_{N} X, P Y\right), & \widetilde{g}\left(A_{N} X, N\right)=0 .
\end{array}
$$

From $(2.11), A_{\xi}^{*}$ is $S(T M)$-valued and self-adjoint on $T M$ such that

$$
A_{\xi}^{*} \xi=0
$$

We denote by $\widetilde{R}, R$ and $R^{*}$ the curvature tensors of the Levi-Civita connection $\widetilde{\nabla}$ of $\widetilde{M}$, the induced connection $\nabla$ of $M$ and the connection $\nabla^{*}$ on $S(T M)$, respectively. Using the Gauss-Weingarten equations for $M$ and $S(T M)$, we obtain the Gauss-Codazzi equations for $M$ and $S(T M)$ such that

(2.14) $\widetilde{g}(\widetilde{R}(X, Y) Z, P W)=g(R(X, Y) Z, P W)$

$$
+B(X, Z) C(Y, P W)-B(Y, Z) C(X, P W),
$$

(2.15) $\widetilde{g}(\widetilde{R}(X, Y) Z, \xi)=\left(\nabla_{X} B\right)(Y, Z)-\left(\nabla_{Y} B\right)(X, Z)$

$$
+B(Y, Z) \tau(X)-B(X, Z) \tau(Y),
$$

(2.16) $\widetilde{g}(\widetilde{R}(X, Y) Z, N)=\widetilde{g}(R(X, Y) Z, N)$,

(2.17) $\widetilde{g}(\widetilde{R}(X, Y) \xi, N)=g\left(A_{\xi}^{*} X, A_{N} Y\right)-g\left(A_{\xi}^{*} Y, A_{N} X\right)-2 d \tau(X, Y)$,

(2.18) $g(R(X, Y) P Z, P W)=g\left(R^{*}(X, Y) P Z, P W\right)$

$$
+C(X, P Z) B(Y, P W)-C(Y, P Z) B(X, P W),
$$

(2.19) $\widetilde{g}(R(X, Y) P Z, N)=\left(\nabla_{X} C\right)(Y, P Z)-\left(\nabla_{Y} C\right)(X, P Z)$

$$
+C(X, P Z) \tau(Y)-C(Y, P Z) \tau(X)
$$

for any $X, Y, Z, W \in \Gamma(T M)$.

The Ricci tensor, denoted by $\widetilde{R i c}$, of $\widetilde{M}$ is defined by

$$
\widetilde{\operatorname{Ric}}(X, Y)=\operatorname{trace}\{Z \rightarrow \widetilde{R}(Z, X) Y\}
$$


for any $X, Y \in \Gamma(T \widetilde{M})$. Let $\operatorname{dim} \widetilde{M}=m+2$. Locally, $\widetilde{R i c}$ is given by

$$
\widetilde{\operatorname{Ric}}(X, Y)=\sum_{i=1}^{m+2} \epsilon_{i} \widetilde{g}\left(\widetilde{R}\left(E_{i}, X\right) Y, E_{i}\right)
$$

where $\left\{E_{1}, \ldots, E_{m+2}\right\}$ is an orthonormal frame field of $T \widetilde{M}$. If

$$
\widetilde{R i c}=\widetilde{\kappa} \widetilde{g}, \quad \widetilde{\kappa} \text { is a constant, }
$$

then $\widetilde{M}$ is an Einstein manifold. The scalar curvature $\widetilde{r}$ is defined by

$$
\widetilde{r}=\sum_{i=1}^{m+2} \epsilon_{i} \widetilde{\operatorname{Ric}}\left(E_{i}, E_{i}\right)
$$

Putting (2.21) in (2.22) implies that $\widetilde{M}$ is Einstein if and only if

$$
\widetilde{R i c}=\frac{\widetilde{r}}{m+2} \widetilde{g}
$$

\section{Tangential curvature vector field}

Let $M$ be a lightlike hypersurface of a semi-Riemannian manifold $\widetilde{M}$ of quasi-constant curvature. We may assume that the curvature vector field $\zeta$ of $\widetilde{M}$ is a unit spacelike vector field and $\operatorname{dim} \widetilde{M}>3$. Consider an induced quasi-orthonormal frame field $\left\{\xi ; W_{a}\right\}$ on $M$, where $T M^{\perp}=\operatorname{Span}\{\xi\}$ and $S(T M)=\operatorname{Span}\left\{W_{a}\right\}$ and let $E=\left\{\xi, N, W_{a}\right\}$ be the corresponding frame field on $\widetilde{M}$. By using (2.20), we get

$$
\begin{aligned}
\widetilde{R i c}(X, Y)= & \sum_{a=1}^{m} \epsilon_{a} \widetilde{g}\left(\widetilde{R}\left(W_{a}, X\right) Y, W_{a}\right) \\
& +\widetilde{g}(\widetilde{R}(\xi, X) Y, N)+\widetilde{g}(\widetilde{R}(N, X) Y, \xi),
\end{aligned}
$$

where $\epsilon_{a}$ denotes the causal character $( \pm 1)$ of respective vector field $W_{a}$. Let $R^{(0,2)}$ denote the induced Ricci type tensor of type $(0,2)$ on $M$ given by

$$
R^{(0,2)}(X, Y)=\operatorname{trace}\{Z \rightarrow R(Z, X) Y\}, \quad \forall X, Y \in \Gamma(T M) .
$$

Using the induced quasi-orthonormal frame field $\left\{\xi ; W_{a}\right\}$ on $M$, we obtain

$$
R^{(0,2)}(X, Y)=\sum_{a=1}^{m} \epsilon_{a} g\left(R\left(W_{a}, X\right) Y, W_{a}\right)+\widetilde{g}(R(\xi, X) Y, N) .
$$

Substituting (2.15) and (2.18) in (3.1) an using (2.12) and (2.13), we obtain

$$
\begin{aligned}
R^{(0,2)}(X, Y)= & \widetilde{\operatorname{Ric}}(X, Y)+B(X, Y) \operatorname{tr} A_{N}-g\left(A_{N} X, A_{\xi}^{*} Y\right) \\
& -\widetilde{g}(R(\xi, Y) X, N), \quad \forall X, Y \in \Gamma(T M) .
\end{aligned}
$$


This shows that $R^{(0,2)}$ is not symmetric. The tensor field $R^{(0,2)}$, defined by (3.2), is called its induced Ricci tensor [5], denoted by Ric, if it is symmetric. Using (2.17), (3.4) and the first Bianchi's identity, we obtain

$$
R^{(0,2)}(X, Y)-R^{(0,2)}(Y, X)=2 d \tau(X, Y), \quad \forall X, Y \in \Gamma(T M) .
$$

Theorem $3.1([4,5])$. Let $M$ be a lightlike hypersurface of a semi-Riemannian manifold $\widetilde{M}$. Then the Ricci type tensor $R^{(0,2)}$ is symmetric if and only if the 1 -form $\tau$ is closed, i.e., $d \tau=0$, on any coordinate neighborhood $\mathcal{U} \subset M$.

In the sequel, we assume that the curvature vector field $\zeta$ of $\widetilde{M}$ is tangent to $M$ and let $e=\theta(N)$. From (1.1), (2.15) and (2.16), we have

$$
\begin{aligned}
\left(\nabla_{X} B\right)(Y, Z)-\left(\nabla_{Y} B\right)(X, Z)=B(X, Z) \tau(Y)-B(Y, Z) \tau(X), & \\
\widetilde{g}(R(X, Y) Z, N)= & \alpha \alpha \eta(X)+e \beta \theta(X)\} g(Y, Z) \\
& -\{\alpha \eta(Y)+e \beta \theta(Y)\} g(X, Z) \\
& +\beta\{\theta(Y) \eta(X)-\theta(X) \eta(Y)\} \theta(Z)
\end{aligned}
$$

for all $X, Y, Z \in \Gamma(T M)$. Using (1.1), (2.20) and (3.6), we have

$$
\begin{aligned}
& \widetilde{\operatorname{Ric}}(X, Y)=\{(m+1) \alpha+\beta\} g(X, Y)+m \beta \theta(X) \theta(Y), \\
& \widetilde{g}(R(\xi, Y) X, N)=\alpha g(X, Y)+\beta \theta(X) \theta(Y), \forall X, Y \in \Gamma(T M) .
\end{aligned}
$$

Substituting this two equations into (3.4), we have

$$
\begin{aligned}
R^{(0,2)}(X, Y)= & \{m \alpha+\beta\} g(X, Y)+\beta(m-1) \theta(X) \theta(Y) \\
& +B(X, Y) \operatorname{tr} A_{N}-g\left(A_{N} X, A_{\xi}^{*} Y\right), \forall X, Y \in \Gamma(T M) .
\end{aligned}
$$

Definition 1. We say that the screen distribution $S(T M)$ of $M$ is totally umbilical [4] in $M$ if, on any coordinate neighborhood $\mathcal{U} \subset M$, there is a smooth function $\gamma$ such that $A_{N} X=\gamma P X$ for any $X \in \Gamma(T M)$, or equivalently,

$$
C(X, P Y)=\gamma g(X, Y), \quad \forall X, Y \in \Gamma(T M) .
$$

In case $\gamma=0$ on $\mathcal{U}$, we say that $S(T M)$ is totally geodesic in $M$.

Using (3.9) and the fact $A_{\xi}^{*}$ is self-adjoint, we have:

Theorem 3.2. Let $M$ be a lightlike hypersurface of a semi-Riemannian manifold $\widetilde{M}$ of quasi-constant curvature. If $S(T M)$ is totally umbilical in $M$ and $\zeta$ is tangent to $M$, then $R^{(0,2)}$ is an induced Ricci tensor Ric of $M$.

Theorem 3.3. Let $M$ be a lightlike hypersurface of a semi-Riemannian manifold $\widetilde{M}$ of quasi constant curvature. If $S(T M)$ is totally geodesic in $M$ and $\zeta$ is tangent to $M$, then the functions $\alpha$ and $\beta$, defined by (1.1), vanish identically. Furthermore, $\widetilde{M}$ and $M$ are flat manifolds.

Proof. As $C=0$, we have $\widetilde{g}(R(X, Y) P Z, N)=0$ due to (2.19). From Theorem 3.1 and Theorem 3.2, we show that $d \tau=0$ on $T M$. Thus we also have $\widetilde{g}(R(X, Y) \xi, N)=0$ due to $(2.17)$. From this two results we get

$$
\widetilde{g}(R(X, Y) Z, N)=0, \quad \forall X, Y, Z \in \Gamma(T M) .
$$


From this equation and the equation (3.8), we have

$$
\beta \theta(X) \theta(Y)=-\alpha g(X, Y), \quad \forall X, Y \in \Gamma(T M) .
$$

Substituting (3.12) into (1.1) and using (2.14), (2.15) and (3.5), we have

$$
R(X, Y) Z=\alpha\{g(X, Z) Y-g(Y, Z) X\}, \quad \forall X, Y, Z \in \Gamma(T M) .
$$

Thus $M$ is a space of constant curvature $-\alpha$. Taking $X=Y=\zeta$ to (3.12), we have $\alpha+\beta=0$. Substituting (3.12) and $A_{N}=0$ into (3.9), we have

$$
\operatorname{Ric}(X, Y)=0, \quad \forall X, Y \in \Gamma(T M) .
$$

Substituting (3.13) and $g(R(\xi, Y) X, N)=0$ into (3.3), we also have

$$
\operatorname{Ric}(X, Y)=-(m-1) \alpha g(X, Y), \quad \forall X, Y \in \Gamma(T M) .
$$

From the last two equations, we obtain $\alpha=0$ as $m>1$. Thus $\beta=0$ and $\widetilde{M}$ and $M$ are flat manifolds, by (1.1) and (3.13).

Corollary 1. There exist no non-flat lightlike hypersurfaces $M$ of semi-Riemannian manifold $\widetilde{M}$ of quasi-constant curvature such that $S(T M)$ is totally geodesic in $M$ and the curvature vector field $\zeta$ of $\widetilde{M}$ is tangent to $M$.

Theorem 3.4. Theorem 3.4. Let $M$ be a lightlike hypersurface of a semiRiemannian manifold $\widetilde{M}$ of quasi constant curvature. If $S(T M)$ is totally umbilical in $\widetilde{M}$ and $\zeta$ is tangent to $M$, then the scaler function $\beta$ vanishes identically. Furthermore, $\widetilde{M}$ is a space of constant curvature $\alpha$ and $M$ is an Einstein manifold such that Ric $=(r / m) g$, where $r$ is the scalar curvature of $M$.

Proof. Assume $S(T M)$ is totally umbilical in $\widetilde{M}$. Then we have (3.10) and

$$
B(X, Y)=\rho g(X, Y), \forall X, Y \in \Gamma(T M) .
$$

Applying $\nabla_{Z}$ to (3.10) and using (2.9), we have

$$
\left(\nabla_{X} C\right)(Y, P Z)=(X \gamma) g(Y, P Z)+\gamma B(X, P Z) \eta(Y)
$$

for all $X, Y, Z \in \Gamma(T M)$. Substituting this into (2.19) and using (3.6), (3.14) and the fact $\theta(\xi)=0$, we obtain

$$
\begin{aligned}
& \{X[\gamma]-\gamma \tau(X)-\rho \gamma \eta(X)-\alpha \eta(X)-e \beta \theta(X)\} g(Y, Z) \\
= & \{Y[\gamma]-\gamma \tau(Y)-\rho \gamma \eta(Y)-\alpha \eta(Y)-e \beta \theta(Y)\} g(X, Z) \\
& +\beta\{\theta(Y) \eta(X)-\theta(X) \eta(Y)\} \theta(Z), \quad \forall X, Y, Z \in \Gamma(T M) .
\end{aligned}
$$

Replacing $Y$ by $\xi$ to this equation and using the fact $\theta(\xi)=0$, we have

$$
\{\xi[\gamma]-\gamma \tau(\xi)-\rho \gamma-\alpha\} g(X, Y)=\beta \theta(X) \theta(Y), \quad \forall X, Y \in \Gamma(T M) .
$$

Taking $X=Y=\zeta$ to this equation, we have $\beta=\xi[\gamma]-\gamma \tau(\xi)-\rho \gamma-\alpha$ and

$$
g(X, Y)=\theta(X) \theta(Y), \quad \forall X, Y \in \Gamma(T M) \text {. }
$$


Substituting (3.15) into (1.1) and using (2.14), (2.15) and (3.14), we have (3.16) $g(R(X, Y) Z, W)=(\alpha+2 \beta+\rho \gamma)\{g(Y, Z) g(X, W)-g(X, Z) g(Y, W)\}$ for all $X, Y, Z, W \in \Gamma(T M)$. Substituting (3.8) and (3.16) into (3.3), we have (3.17) $\operatorname{Ric}(X, Y)=\{m \alpha+(2 m-1) \beta+(m-1) \rho \gamma) g(X, Y), \forall X, Y \in \Gamma(T M)$.

On the other hand, substituting (3.15) into (3.9) and using the facts $\operatorname{tr} A_{N}=m \gamma$ and $g\left(A_{\xi}^{*} X, A_{N} Y\right)=\rho \gamma g(X, Y)$, we have

$$
\operatorname{Ric}(X, Y)=\{m(\alpha+\beta)+(m-1) \rho \gamma\} g(X, Y), \forall X, Y \in \Gamma(T M) .
$$

Comparing (3.17) and (3.18), we obtain

$$
(m-1) \beta=0 \text {. }
$$

As $m>1$, we have $\beta=0$. Thus $M$ is a space of constant curvature $\alpha$.

Let $\kappa=m \alpha+(m-1) \rho \gamma$. Then the equations (3.17) and (3.18) reduce to

$$
\operatorname{Ric}(X, Y)=\kappa g(X, Y), \forall X, Y \in \Gamma(T M) .
$$

Thus $M$ is an Einstein manifold. The scalar quantity $r$ [3] of $M$, obtained from $R^{(0,2)}$ by the method of $(2.22)$ is given by

$$
r=R^{(0,2)}(\xi, \xi)+\sum_{a=1}^{m} \epsilon_{a} R^{(0,2)}\left(W_{a}, W_{a}\right) .
$$

Since $M$ is an Einstein manifold satisfying (3.19), we obtain

$$
r=\kappa g(\xi, \xi)+\kappa \sum_{a=1}^{m} \epsilon_{a} g\left(W_{a}, W_{a}\right)=\kappa m .
$$

Thus we have

$$
\operatorname{Ric}(X, Y)=(r / m) g(X, Y)
$$

which provides a geometric interpretation of lightlike Einstein hypersurfaces (same as in Riemannian case) as we have shown that the constant $\kappa=r / m$.

\section{References}

[1] M. C. Chaki and R. K. Maity, On quasi-Einstein manifolds, Publ. Math. Debrecen 57 (2000), no. 3-4, 297-306.

[2] B. Y. Chen and K. Yano, Hypersurfaces of a conformally flat space, Tensor (N. S.) 26 (1972), 318-322.

[3] K. L. Duggal, On scalar curvature in lightlike geometry, J. Geom. Phys. 57 (2007), no. 2, 473-481.

[4] K. L. Duggal and A. Bejancu, Lightlike Submanifolds of Semi-Riemannian Manifolds and Applications, Kluwer Acad. Publishers, Dordrecht, 1996.

[5] K. L. Duggal and D. H. Jin, Null curves and Hypersurfaces of Semi-Riemannian Manifolds, World Scientific, 2007.

[6] — A classification of Einstein lightlike hypersurfaces of a Lorentzian space form, J. Geom. Phys. 60 (2010), no. 12, 1881-1889. 
Department of Mathematics

DONGGUK UNIVERSITY

GyeonguU 780-714, KoreA

E-mail address: jindh@dongguk.ac.kr 